\section{Dietary microbial toxins and Type 1 diabetes - a new meaning for seed and soil}

To the Editor: There are acknowledged environmental contributions to Type I (insulin-dependent) diabetes mellitus and viruses, whereby cows' milk proteins and dietary toxins are suspected [1]. We have found in mice that nanogram quantities of the macrolide antibiotic bafilomycin A1 cause glucose intolerance and pancreatic islet disruption. Bafilomycin A1 and related macrolides are produced by Streptomyces species ubiquitous in soil. These species can infest tuberous vegetables, particularly potatoes and beet [2-4]. Hence dietary exposure to these macrolides could damage pancreatic islets and thus cause diabetes in genetically susceptible subjects.

The islet beta cell is highly susceptible to metabolic stress, free radicals and cytokine exposure $[5,6]$. In repeated sub-toxic doses, the beta-cell specific toxin, streptozotocin, induces autoimmune diabetes in certain inbred strains of rodents [7], showing that toxic damage can cause release and/or modification of beta-cell constituents and elicitation of autoimmunity. Notably, streptozotocin is derived from Streptomyces spp.

We carried out experiments in mice to examine if the vacuolar ATPase could be involved in the failure of insulin secretion. The experiments followed the principles of laboratory animal care (NIH publication No. 86-23, revised 1985). Islets proved particularly sensitive to the highly specific vATPase inhibitor, bafilomycin A1. Male BALB/c mice injected intraperitoneally with $12 \mu \mathrm{g}$ per kilogram body weight of bafilomycin A1 (Sigma, St. Louis, Mo., USA), which inhibited kidney vATPase enzyme activity by $50 \%$, showed glucose intolerance that progressively worsened. For six mice treated 21 days previously with bafilomycin A1 the peak blood glucose concentration $15 \mathrm{~min}$ after glucose challenge was $12.5 \pm 2.4 \mathrm{mmol} / \mathrm{l} \mathrm{com}-$ pared with $9.5 \pm 1.8 \mathrm{mmol} / \mathrm{l}$ for 18 control mice $(p=0.04$, Student's $t$ test). There was preservation of normal fasting glucose despite glucose intolerance, suggesting impairment of firstphase insulin release. For eight mice studied 7 days after receiving bafilomycin A1 fasting concentrations of insulin and/ or proinsulin (sensitive rat insulin RIA, Linco, St. Charles, MO) were lower than in the control mice $(1.2 \pm 0.8$, vs $3.1 \pm 1.3 \mathrm{ng} / \mathrm{ml}, p=0.004$, Student's $t$ test). Fifteen minutes after a glucose challenge plasma insulin concentrations were similar. Pancreata from five treated mice contained $70 \%$ more cellular insulin and/or proinsulin than four control mice $($ median $=565$, range $287-718$ vs median $=273$, range $159-453 \mu \mathrm{g}$ immunoreactive insulin per gram of pancreas, $p<0.03$, Mann-Whitney U test). Effects of bafilomycin A1 on islet morphology were examined in mice 90 days after two weekly injections of bafilomycin A1. Islet size measured morphometrically on histological sections was markedly reduced in treated mice (Fig. 1) ( $p=0.0003$, Mann-Whitney U test). Kidney, liver, testes, and brain were normal.

The noticeable effects of bafilomycin A1 on insulin secretion, glucose tolerance and islet structure prompted the question of whether toxic exposure to bafilomycin A1 and related compounds could occur naturally. Bafilomycin A1 was originally isolated from $S$. griseus, a common soil microbe known to infest tuberous vegetables, notably potatoes and beet [4]. The diversity of Streptomyces spp. capable of infesting vegetables $[2,3]$ and their potential to produce toxic secondary metabolites such as streptozotocin and bafilomycin A1 provide a likely avenue for beta-cell toxins to enter the human diet. Whilst intraperitoneal administration was used to ensure uniformity of dosage, bafilomycin A1 would be expected to transfer across the intestine since non-toxic macrolides, such as control

treated
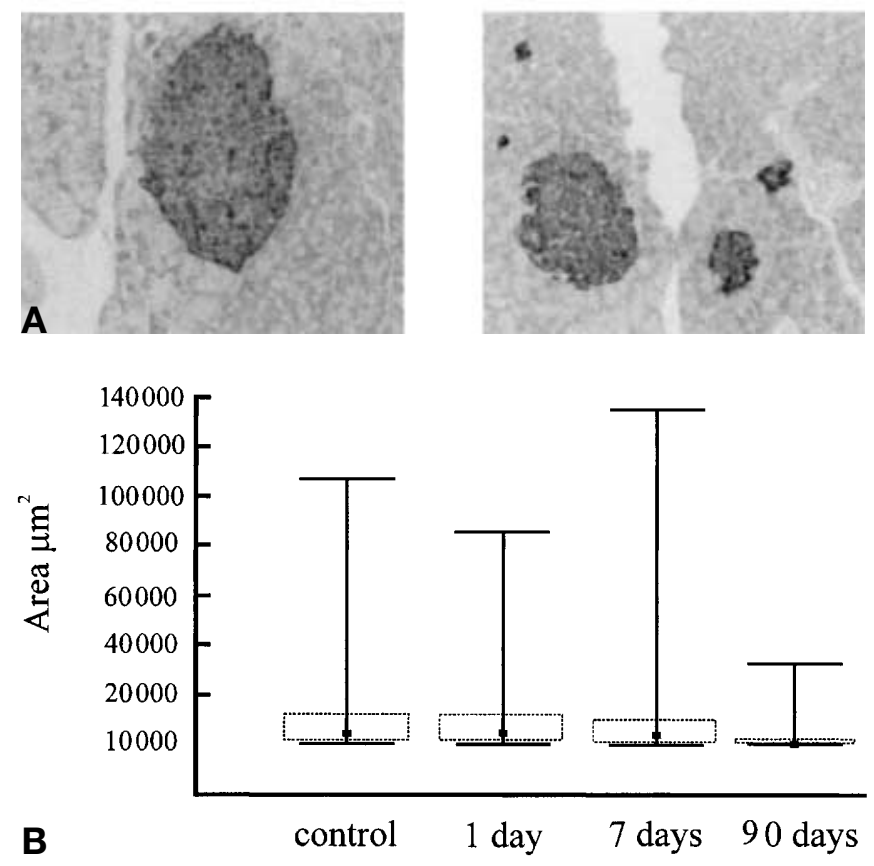

Fig. 1(A, B). The islets from bafilomycin A1-treated mice are reduced in size. A Sections of mouse pancreas immunostained for insulin and counterstained with haematoxylin showing an islet from a control mouse and smaller, disrupted islets from a bafilomycin A1 treated mouse. B Islet size was determined morphometrically on sections stained with insulin antibody for controls ( 145 islets from eight mice) or 1 day ( 81 islets from three mice), 7 days (125 islets from three mice) or 90 days (81 islets from four mice) after receiving bafilomycin A1 using digitised images and MCID software. The black box (ם) represents the median, dotted boxes ( I ) the 25 th -75 th centiles and the lines maximum and minimum value

erythromycin, are used orally. We note that Type I diabetes has a high prevalence in Western countries where consumption of tuberous vegetables and sugar refined from beets is common. The knowledge that bafilomycin A1 and related macrolide antibiotics damage beta islet cells in mice and are produced by soil-dwelling Streptomyces spp. that contaminate dietary vegetables is a novel lead to environmental causes of Type I diabetes.

M. A. Myers, I. R. Mackay, M. J. Rowley, P.Z.Zimmet

\section{References}

1. Akerblom HK, Knip M (1998) Putative environmental factors in Type 1 diabetes. Diabetes Metab Rev 14: 31-67

2. Doering-Saad C, Kampfer P, Manulis S et al. (1992) Diversity among Streptomyces Strains causing Potato Scab. Appl and Environ Microbiol 58: 3932-3940

3. Archuleta JG, Easton GD (1981) The cause of deep pitted scab of potatoes. Am Potato J 58: 385-392

4. Loria R, Bukhalid RA, Fry BA, King RR (1997) Plant Pathogenicity in the Genus Streptomyces. Plant Disease 81: 836-846 
5. Rabinovitch A (1998) An update on cytokines in the pathogenesis of insulin-dependent diabetes mellitus Diabetes Metab Rev 14: 129-151

6. Amrani A, Verdaguer J, Thiessen S, Bou S, Santamaria P (2000) IL-1alpha, IL-1beta, and IFN-gamma mark beta cells for Fas-dependent destruction by diabetogenic CD4( + ) T lymphocytes. J Clin Invest 105: 459-468
7. Like A, Rossini A (1976) Streptozotocin-induced pancreatic insulitis: new model of diabetes mellitus. Science 193: 415-417

Corresponding author: MA Myers, Department of Biochemistry and Molecular Biology, Monash University, Wellington Road, Clayton, Victoria, 3800, Australia, E-mail: Mark. Myers@med.monash.edu.au

\section{G972R polymorphism of IRS-1 in women with polycystic ovary syndrome}

To the Editor: Studies have reported a link between insulin resistance and polycystic ovary syndrome, one of the most common endocrine disorders of premenopausal women, characterized by anovulation and hyperandrogenism. Insulin resistance with increased risk of Type II (non-insulin-dependent) diabetes mellitus is a common comorbidity in patients with polycystic ovary syndrome which involves a post-binding defect in insulin receptor signalling [1]. In at least $50 \%$ of the women with polycystic ovary syndrome, insulin resistance could be related to reduced tyrosine kinase activity and excessive serine phosphorylation of the insulin receptor [2]. In the other $50 \%$, the pathogenesis of insulin resistance is still not known.

Insulin receptor substrate (IRS)-1 is the major cytoplasmic substrate of the insulin receptor. Many polymorphisms have been described in IRS-1, the most common aminoacid change being a glycine to arginine substitution at codon 972 (G972R) [3], which has an overall frequency of $5.8 \%$ in the general population and is the most common variant observed in Type II diabetes [4]. Transfection studies indicate that this variant could impair insulin-stimulated signalling, especially along the phosphatidylinositol 3-kinase pathway which controls glucose transport [5]. In human beings, this genetic variant is associated with reduced insulin sensitivity and with obesity and body fat distribution $[6,7]$.

We therefore examined the association between IRS-1 G972Arg genotype and PCOS and the metabolic characteristics of overweight (BMI $\geq 25 \mathrm{~kg} / \mathrm{m}^{2}$ ) (WHO criteria) and normal weight $\left(\mathrm{BMI}<24.9 \mathrm{~kg} / \mathrm{m}^{2}\right.$ ) polycystic ovary syndrome patients who were carriers of codon-972 variant (PCOS carriers). In 82 PCOS patients ( 23 normal weight and 59 overweight) and in 70 normal women (21 normal weight and 49 overweight), G972R genotypes were obtained by PCR amplification and BstN-1 restriction enzyme digestion [7]. An OGTT was done in all patients. Serum glucose and insulin were measured before the glucose load and 60 and 120 min after. Ho- meostasis model assessment for insulin-resistance $\left(\mathrm{HOMA}_{\mathrm{IR}}\right)$ and whole-body insulin sensitivity (ISI composite) were calculated [8]. Glucose was determined by the glucose oxidase method and insulin was assayed by RIA (DPC, Los Angeles, Calif., USA). All patients had given their written consent to their participation in the study which was approved by the local ethics committee. Statistical analysis was performed using chi square $\left(\chi^{2}\right)$ and Mann-Whitney tests. Differences were considered significant at $p<0.05$.

The frequency of $G 972 R$ variant (0.121) was higher $(p<0.02)$ in PCOS patients compared to normal women $(0.055)$.

Post-stimulated 2-h insulin was higher in PCOS carriers of codon-972 than in those without the mutation (Table 1). Among the PCOS carriers, six were overweight and four had normal weight. When normal weight and overweight carriers were compared to normal weight $[97.6(17.3 ; 176.7)$ vs 72.2 $(9.4 ; 251.8) \mu \mathrm{UI} / \mathrm{ml}]$ and overweight [178.(140.8;268.7) vs 97.2 $(25.1 ; 296.1) \mu \mathrm{UI} / \mathrm{ml}]$ patients without this mutation, post-stimulated 2-h insulin remained higher in the PCOS carriers. HO$\mathrm{MA}_{\mathrm{IR}}$ and ISI composite in normal weight and overweight PCOS carriers were 1.97 vs $6.03(p=0.038)$ and 4.41 vs 1.71 $(p=0.020)$, respectively.

These data suggest that the frequency of the heterozygote form of codon -972 variant is higher in PCOS patients than in normal women. Differences in $\mathrm{HOMA}_{\mathrm{IR}}$ and ISI composite between normal weight and overweight carriers, indicate that this IRS-1 variant interacts with obesity to influence insulin resistance, with little effect if the weight is normal, as previously suggested [7].

Acknowledgements. This work was supported by a grant from Fondecyt (1000973) and by the Alexander von Humboldt Foundation.

T.Sir-Petermann, F.Pérez-Bravo, B. Angel, M. Maliqueo, M. Calvillan, A. Palomino

Table 1. Metabolic parameters in PCOS patients according to the $G 972 R$ variant $^{\mathrm{a}}$

\begin{tabular}{llllll}
\hline & Insulin $(\mu \mathrm{UI} / \mathrm{ml})$ & & & & \\
& Fasting & $60 \mathrm{~min}$ & $120 \mathrm{~min}$ & HOMA $_{\text {IR }}$ & ISI composite \\
\hline PCOS (Gly/Gly) & 20.56 & 102.89 & 85.95 & 4.31 & 2.58 \\
$(n=72)$ & $(4.1-53.7)$ & $(28.8-355.4)$ & $(9.4-296.1)$ & $(0.6-13.6)$ & $(1.05-18.89)$ \\
PCOS (Gly/Arg) & 19.88 & 173.35 & 166.30 & 4.66 & 2.16 \\
$(n=10)$ & $(3.74-41.2)$ & $(60.2-269.0)$ & $(17.3-268.7)^{*}$ & $(0.7-9.0)$ & $(1.34-8.98)$ \\
\hline
\end{tabular}

$* p<0.05$ between PCOS(Gly/Gly) v/s PCOS(Gly/Arg)

${ }^{\mathrm{a}}$ Values are median and range 